\title{
Characteristics of Sepsis-2 septic shock patients failing to satisfy the Sepsis-3 septic shock definition: an analysis of real-time collected data
}

\author{
Joris Vermassen ${ }^{1 *}$, Johan Decruyenaere ${ }^{1,2}$, Liesbet De Bus ${ }^{1}$, Pieter Depuydt ${ }^{1,2}$ and Kirsten Colpaert ${ }^{1,2}$
}

\begin{abstract}
Background: Baseline characteristics and disease severity of patients with septic shock according to the new Sepsis-3 definition may differ from patients that only comply with the Sepsis-2 definition. We conducted a retrospective cohort study on the ICU of a Belgian tertiary care facility to seek out differences between these two patient groups and to identify variables associated with no longer satisfying the latest definition of septic shock.

Results: Of 1198 patients with septic shock according to the Sepsis-2 consensus definition, 233 (19.4\%) did not have septic shock according to the Sepsis-3 shock definition. These patients more often had medical admission reasons and a respiratory infection as cause for the septic shock. They less often had surgery on admission and were less likely to have chronic liver disease (5.6\% vs 16.2\%, absolute difference 10.6\% (95\% Cl 6.4-14.1\%). Patients with septic shock only according to the old definition had significant lower APACHE II and SOFA scores and lower hospital mortality (31.6\% vs $55.3 \%, p<0.001)$. In a multivariate analysis, following variables were associated with Sepsis-2 shock patients no longer being defined as such by the Sepsis-3 definition: respiratory infection (OR 1.485 ( $95 \% \mathrm{Cl} 1.56-2.089)$, $p=0.023)$, a medical admission reason (OR 1.977 (95\% Cl 1.396-2.800) and chronic liver disease (OR 0.345 (95\% Cl $0.181-0.660), p<0.001)$

Conclusions: One in five patients with septic shock according to the Sepsis-2 consensus definition is no longer considered as such when the Sepsis-3 shock criteria are applied. A medical admission reason, a respiratory infection and absence of chronic liver disease are independently associated with no longer being identified as having septic shock by the Sepsis-3 criteria.
\end{abstract}

Keywords: Septic shock, Intensive care unit, Epidemiology, Lactate, Chronic liver disease

\section{Background}

In 2016 a new consensus definition of sepsis and septic shock was published. Patients are required to have sepsis (a proven or suspected infection in combination with a rise in sequential organ failure assessment score (SOFA) of at least 2 points compared to baseline), persistent

\footnotetext{
*Correspondence: Joris.vermassen@uzgent.be

${ }^{1}$ Department of Intensive Care Medicine, University Hospital Gent,

2K12C. C. Heymanslaan 10, 9000 Gent, Belgium

Full list of author information is available at the end of the article
}

hypotension requiring vasopressor therapy to maintain a mean arterial pressure of at least $65 \mathrm{~mm}$ of mercury $(\mathrm{Hg})$ and a lactate of at least $2 \mathrm{mmol} / \mathrm{l}$ despite adequate volume resuscitation to be considered to have septic shock according to this new consensus definition [1]. In contrast, for patients to comply with the previous Sepsis-2 consensus definition, they needed to have sepsis (defined as a proven or suspected infection in combination with at least 2 systemic inflammatory response syndrome (SIRS) criteria) and persistent hypotension (defined as a

\section{Springer Open}

(c) The Author(s) 2021. Open Access This article is licensed under a Creative Commons Attribution 4.0 International License, which permits use, sharing, adaptation, distribution and reproduction in any medium or format, as long as you give appropriate credit to the original author(s) and the source, provide a link to the Creative Commons licence, and indicate if changes were made. The images or other third party material in this article are included in the article's Creative Commons licence, unless indicated otherwise in a credit line to the material. If material is not included in the article's Creative Commons licence and your intended use is not permitted by statutory regulation or exceeds the permitted use, you will need to obtain permission directly from the copyright holder. To view a copy of this licence, visit http://creativecommons.org/licenses/by/4.0/. 
mean arterial pressure below $60 \mathrm{~mm} \mathrm{Hg}$, a systolic blood pressure below $90 \mathrm{~mm} \mathrm{Hg}$ or a decrease in systolic blood pressure of at least $40 \mathrm{~mm} \mathrm{Hg}$ ) despite adequate fluid resuscitation [2]. Ever since, the effect of the change of the definition on patient selection has been scrutinized. Some authors report lower sensitivity but higher specificity in detecting patients with septic shock when the new criteria are applied, as well as higher mortality in patients they identify $[3,4]$. The use of SOFA or quick SOFA (qSOFA) also results in a more accurate prediction of risk of mortality compared to the old definitions $[5,6]$. Consequently, a certain number of patients being classified as having sepsis or septic shock according to this old definition will no longer be held as such by the new one [7]. This could result in a subgroup of septic patients no longer receiving the official label "septic shock" and consequently being excluded from clinical trials or epidemiological studies with all consequences thereof. Our primary goal was to investigate baseline characteristics of septic shock patients who meet the old Sepsis- 2 consensus definition but are no longer considered as such by the new Sepsis-3 septic shock definition-with lactate values below $2 \mathrm{mmol} / \mathrm{l}$-and to describe differences in severity of illness. Additionally, we wanted to identify the factors that are associated with this exclusion.

\section{Methods}

This study was performed at the Ghent University Hospital, a tertiary care facility with a 66-bed intensive care unit (ICU) containing a surgical, cardiothoracic, medical, pediatric and burn unit. During the study period, our ICU was $24 / 7$ covered by at least one consultant intensive care physician and four intensive care residents. All infections in our ICU were prospectively recorded in our own ICU's COSARA database. In COSARA (Computer-based Surveillance and Alerting of infections, Antimicrobial Resistance and Antibiotic consumption in the ICU) every infection was graded in severity according to the Sepsis-2 consensus definition $[2,8,9]$. Patients with septic shock were treated according to the surviving sepsis guidelines with norepinephrine as first choice vasopressor [10]. Data from all consecutively admitted patients between January 1st, 2013, and December 31st, 2018, with a diagnosis of septic shock according to COSARA and for whom a prescription for the administration of norepinephrine could be retrieved from the health record, were gathered. We only included patients admitted to the surgical or medical ICU. Patients without reported lactate value were excluded. If patients experienced a recurrent septic shock episode during the same hospitalization, only the first ICU admission was included. The remaining patients were considered to have septic shock according to the Sepsis-2 definition.
Starting from all patients in our database complying with the definition of septic shock according to the Sepsis-2 definition, we created two groups. The first group of patients was called "S3+". These patients had, on top of vasopressor therapy, also a rise in lactate above $2 \mathrm{mmol} / \mathrm{l}$ at the same timepoint, which caused them to comply with the Sepsis-3 shock definition. The remaining patients only had vasopressor therapy, but had no reported lactate value above $2 \mathrm{mmol} / \mathrm{l}$, so they did not satisfy the Sepsis-3 shock definition. These patients were called "S2+/S3- " [1].

Demographic data, comorbidities, infection characteristics, laboratory results, medication prescriptions, organ support variables and outcome parameters were collected from the electronic patient record (GE Healthcare, Centricity Critical Care). Charlson comorbidity index (CCI), SOFA score and Acute Physiology and Chronic Health Evaluation (APACHE II) score were calculated for every patient [11-13]. A medical admission reason was defined as a condition with need for admission in the ICU in patients with otherwise no present indication for surgical intervention.

Numeric variables are expressed as median and interquartile range, nominal variables are expressed as $n(\%)$. Differences between the S3+and S2+/S3- patients were assessed using Chi-square and Mann-Whitney $\mathrm{U}$ test where appropriate and statistical significance was attained at $p<0.05$. Absolute differences between the two defined populations were calculated using the independent samples proportions test. A multivariate logistic regression model was constructed to identify variables independently associated with being excluded from the Sepsis-3 definition of septic shock. Therefore, all variables that were univariately associated with being excluded from the Sepsis-3 septic shock definition were used. Organ dysfunction variables were not used because we aimed to make a baseline risk analysis based only on variables that are possibly known at the moment of the onset of septic shock, in essence when the decision to start supportive therapy should be made.

All analyses were performed with IBM SPSS statistics $\mathrm{v} 26$. Approval for this study was obtained from the local hospital's ethical committee (EC/2016/0254) and the need for informed consent was waived.

\section{Results}

During the 6-year timeframe, 6676 infection episodes requiring antibiotic treatment were registered in COSARA. After exclusion of recurrent episodes of septic shock and of patients missing lactate levels or details on vasopressor therapy, 1198 patients were identified with septic shock according to the Sepsis-2 definition. A total of 233 patients (19.4\%) did not have septic shock 
according to the Sepsis-3 definition (S2+/S3-), 965 patients $(80.6 \%)$ had septic shock both according to the Sepsis-2 and Sepsis-3 definition (S3+) (Fig. 1).

Baseline characteristics of the patients can be found in Table 1. S2+/S3- patients more frequently had a medical admission reason and less surgery on admission. As to infection characteristics, S2+/S3- patients had more respiratory infections causing septic shock, less bacteremia or positive cultures from other clinically significant sites. There was a notable difference in chronic liver disease (CLD) between the S2+/3- and S3+ patients (13 (5.6\%) vs 153 (16.2\%), absolute difference 10.6\% (95\% CI 6.4-14.1\%). Furthermore, patients from the S2+/S3group were more frequently active smokers and were

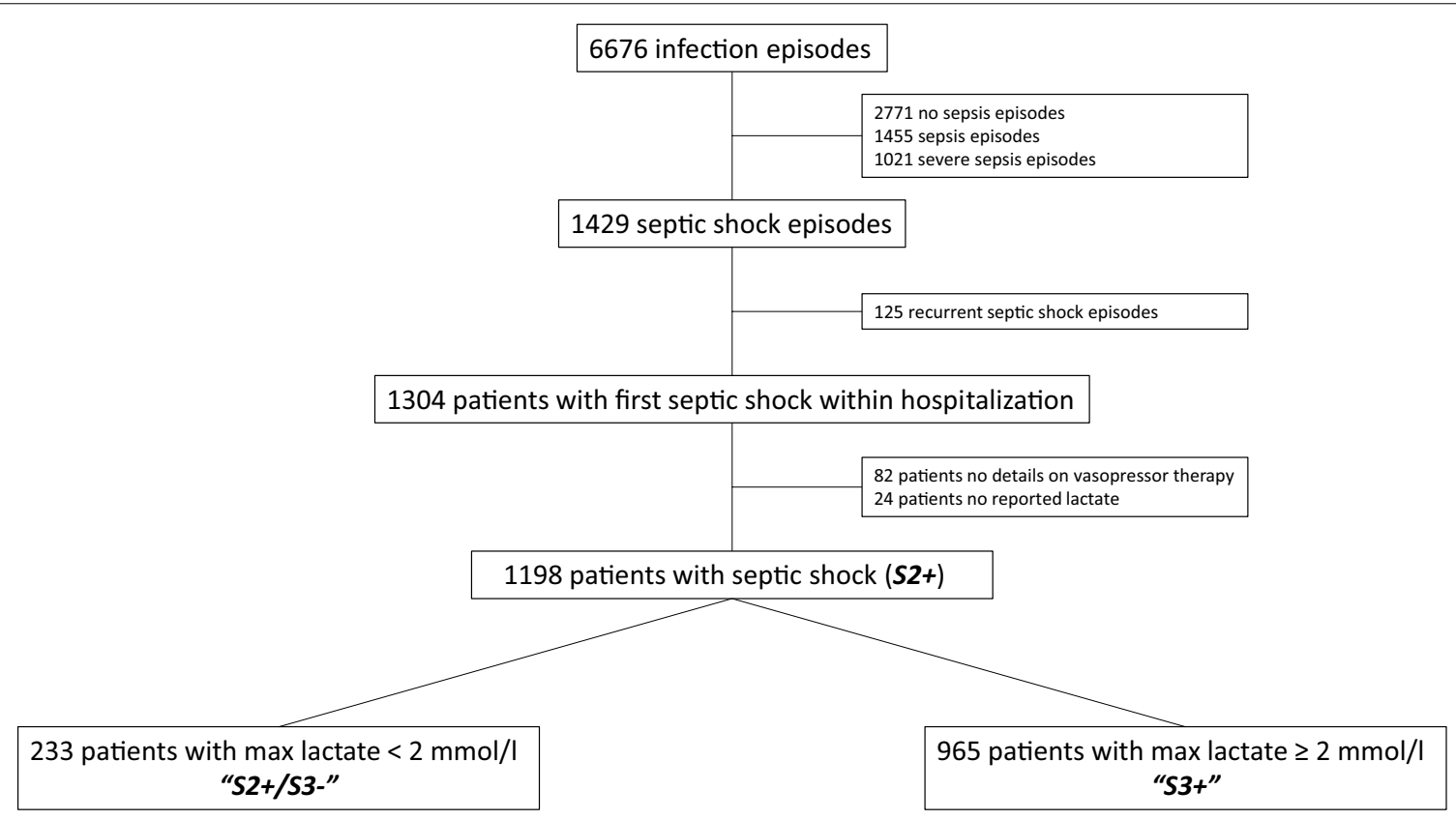

Fig. 1 Patient selection

Table 1 Baseline characteristics of the study population

\begin{tabular}{|c|c|c|c|c|c|c|}
\hline & \multirow[t]{2}{*}{ S3+ } & \multirow[t]{2}{*}{$\mathrm{S} 2+/ \mathrm{S} 3-$} & \multirow{2}{*}{$\begin{array}{l}\text { Absolute } \\
\text { difference }\end{array}$} & \multicolumn{2}{|l|}{$95 \% \mathrm{Cl}$} & \multirow[t]{2}{*}{$p$} \\
\hline & & & & Lower bound & Upper bound & \\
\hline Male & $601(62.3)$ & $160(68.7)$ & -6.4 & -12.9 & 0.4 & 0.069 \\
\hline Age & $64(54-73)$ & $64(54-71)$ & & & & 0.818 \\
\hline Weight & $75(65-85)$ & $76(65-87)$ & & & & 0.095 \\
\hline Living at home & $719(83.7)$ & $172(83.1)$ & 0.6 & -4.8 & 6.5 & 0.832 \\
\hline Admission & & & & & & 0.384 \\
\hline Other hospital & $179(19.0)$ & $36(15.7)$ & & & & \\
\hline Emergency department & $348(37.0)$ & $94(41.0)$ & & & & \\
\hline Ward & $413(43.9)$ & $99(43.2)$ & & & & \\
\hline Readmission $<48 \mathrm{~h}$ & $22(2.3)$ & $9(3.9)$ & -1.6 & -4.6 & 0.9 & 0.172 \\
\hline Medical admission reason & $466(48.3)$ & $155(66.5)$ & -18.2 & -24.9 & -11.3 & $<0.001$ \\
\hline Surgery & $243(25.3)$ & $36(15.5)$ & 9.8 & 4.1 & 14.9 & 0.002 \\
\hline Respiratory septic shock & $287(29.7)$ & $96(41.2)$ & -11.5 & -18.4 & -4.6 & 0.001 \\
\hline Abdominal septic shock & $312(32.3)$ & $45(19.3)$ & 13.0 & 6.9 & 18.7 & $<0.001$ \\
\hline Bacteremia & $262(27.2)$ & $43(18.5)$ & 8.7 & 2.8 & 14.2 & 0.006 \\
\hline Culture negative septic shock & $362(37.5)$ & $110(47.2)$ & -9.7 & -16.8 & -2.6 & 0.007 \\
\hline
\end{tabular}

All values are expressed as median (interquartile range) or $n(\%)$. Absolute differences are reported for percentages 
associated more often with a history of hematological conditions or chronic pulmonary disease and had fewer malignancies (Table 2). Overall comorbidity burden was high with a median CCI of 4 both in the S3+and S2+I S3- group.

Details on organ support and severity of illness can be found in Fig. 2. Fewer patients in the $\mathrm{S} 2+/ \mathrm{S} 3-$ group received mechanical ventilation (MV) (124 (53.2\%) vs 689 (71.4\%), absolute difference $18.2 \%$ (95\% CI 11.2-25.2\%), $p<0.001)$, received renal replacement therapy (RRT) (27 (11.6\%) vs 227 (23.5\%), absolute difference $11.9 \%$ (95\% CI 6.7-16.6\%), $p<0.001)$ or received triple organ support with a combination of vasopressor therapy, MV and RRT (23 (9.9\%) vs 198 (20.5\%), absolute difference 10.6\% (95\% CI 5.7-15.0\%), $p<0.001$ ) compared to S3+ patients. Additionally, they received less adjuvant vasopressor or inotropic support on top of norepinephrine, compared to $\mathrm{S} 3+$ patients. Maximal SOFA score in the $\mathrm{S} 2+/ 3+$ group was $10(8-12.5)$ compared to 13 (10-16) in S3+ patients $(p<0.001)$ and APACHE II score was $24(18-30)$ and 29 (21-35), respectively. A detailed description of organ dysfunction variables can be found in Additional file 1: Tables S4 and S5 as well as in Additional file 1: Fig. S2.

In a multivariate analysis, following criteria were identified with being excluded from the Sepsis-3 septic shock definition (S2+/S3- patients): respiratory infection as cause for septic shock (OR 1.485 (1.0562.089) $p=0.023$ ), medical admission reason (OR 1.977
$(1.396-2.800) p<0.001)$. A comorbidity of chronic liver disease was inversely associated (OR $0.345(0.181-0.660)$ $p<0.001$ ) (Table 3).

ICU and hospital mortality were significantly lower in the S2+/S3 - group (43 (19.4\%) and 65 (31.6\%) vs 416 $(43.9 \%)$ and 501 (55.3\%), $p<0.001)$. In the baseline cohort of patients complying with the Sepsis-2 consensus definition, hospital mortality in patients with CLD was $71.4 \%$, compared to $47.2 \%$ in patients without $(p<0.001)$. In patients with CLD, mortality is higher if they comply with the Sepsis-3 definition than if they only comply with the Sepsis- 2 definition $(75.0 \%$ vs $30.8 \%, p<0.001)$ (Additional file 1: Tables S8 and S9).

\section{Discussion}

We compared characteristics of patients who met the Sepsis-2 shock criteria but not the Sepsis-3 shock definition (S2+/S3-) with those that complied with both septic shock definitions (S3+). In our cohort, 19.4\% of septic shock patients meeting the Sepsis-2 definition did not comply with the Sepsis-3 shock definition. This number is lower than figures reported by other authors [14-18]. A possible explanation is that our patient selection was based on the labeling of septic shock in the COSARA registry, which also necessitates the administration of norepinephrine, whereas other publications have larger proportions of septic shock patients who did not receive vasopressor therapy [15].

Table 2 Comorbidities of the study population

\begin{tabular}{|c|c|c|c|c|c|c|}
\hline & \multirow[b]{2}{*}{ S3+ } & \multirow[b]{2}{*}{$\mathrm{S} 2+/ \mathrm{S} 3-$} & \multirow[b]{2}{*}{$\begin{array}{l}\text { Absolute } \\
\text { difference }\end{array}$} & \multicolumn{2}{|l|}{$95 \% \mathrm{Cl}$} & \multirow[t]{2}{*}{$p$} \\
\hline & & & & Lower bound & Upper bound & \\
\hline Nicotine & $194(25.5)$ & $67(33.8)$ & -8.3 & -15.7 & -1.1 & 0.019 \\
\hline Alcohol abuse & $152(18.5)$ & $23(11.3)$ & 7.2 & 1.8 & 12.0 & 0.014 \\
\hline NYHA III/IV & $242(26.6)$ & $70(31.3)$ & -4.7 & -11.5 & 1.9 & 0.159 \\
\hline Organ transplantation & $19(5.1)$ & $8(10.5)$ & -5.4 & -13.6 & 1.3 & 0.074 \\
\hline Stem cell transplantation & $7(1.9)$ & $0(0)$ & 1.9 & -2.0 & 3.8 & 0.226 \\
\hline Malignancy & $174(28.9)$ & $50(21.6)$ & 7.3 & 1.0 & 13.1 & 0.026 \\
\hline Metastatic malignancy & $100(10.6)$ & $18(7.8)$ & 2.8 & -1.5 & 6.5 & 0.209 \\
\hline Hematologic condition & $111(11.7)$ & $41(17.7)$ & -6.1 & -11.6 & -0.9 & 0.013 \\
\hline Chronic Liver disease & $153(16.2)$ & $13(5.6)$ & 10.6 & 6.4 & 14.1 & $<0.001$ \\
\hline Pulmonary disease & $162(17.2)$ & $54(23.3)$ & -6.1 & -12.2 & -0.3 & 0.032 \\
\hline Chronic kidney disease & $206(21.7)$ & $63(27.3)$ & -5.6 & -12.0 & 0.6 & 0.071 \\
\hline Patient on dialysis & $24(2.5)$ & $11(4.8)$ & -2.2 & -5.5 & 0.5 & 0.073 \\
\hline Diabetes mellitus & $202(21.2)$ & $48(20.7)$ & 0.5 & -5.5 & 6.2 & 0.854 \\
\hline Hypertension & $305(32.2)$ & 78 (33.8) & -1.6 & -8.5 & 5.1 & 0.643 \\
\hline Vascular disease & $160(16.9)$ & $45(19.5)$ & -2.6 & -8.4 & 2.8 & 0.349 \\
\hline Ischemic stroke & $50(5.3)$ & $11(4.8)$ & 0.5 & -3.0 & 3.4 & 0.753 \\
\hline Coronary disease & $180(19.1)$ & 45 (19.4) & -0.3 & -6.2 & 5.2 & 0.909 \\
\hline
\end{tabular}



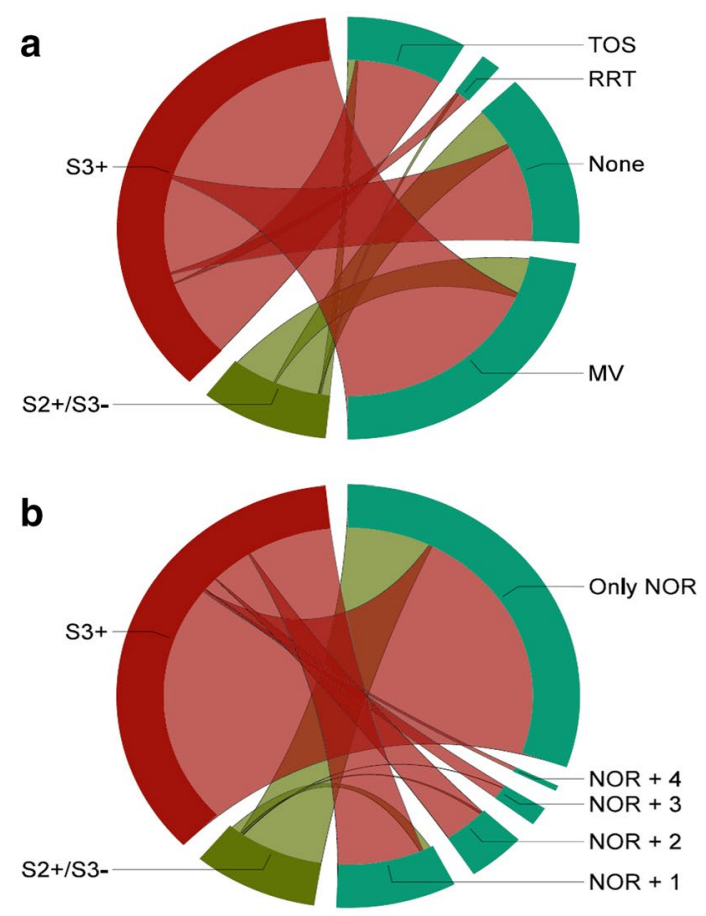
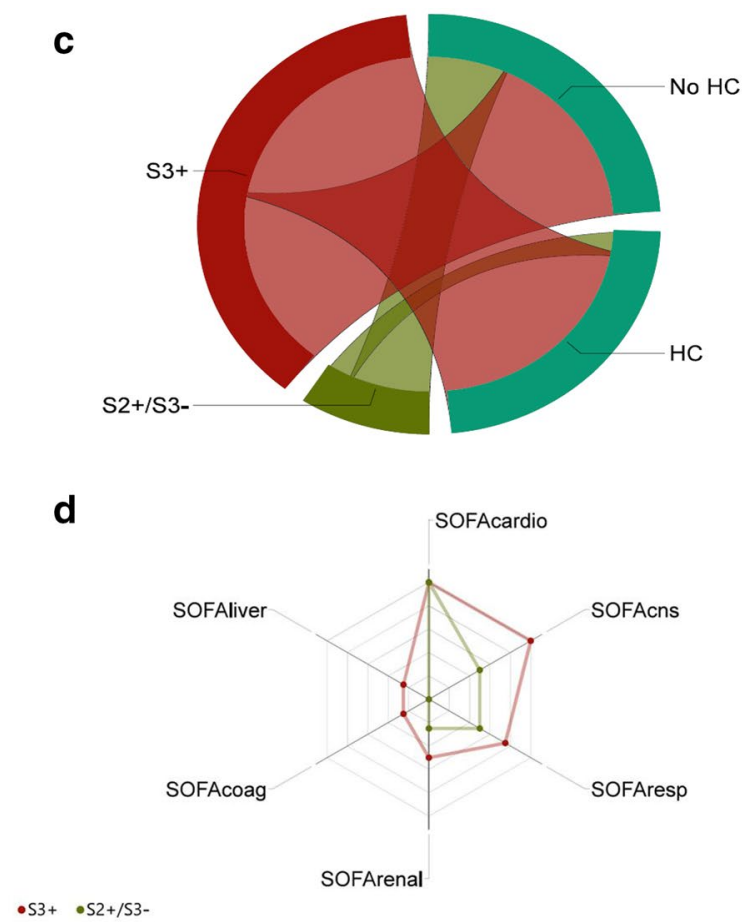

Fig. 2 Differences in organ support and organ dysfunction variables between the $\mathrm{S} 3+$ and $\mathrm{S} 2+/ \mathrm{S} 3-$ patients. a Chord diagram representing the need for organ support - on top of vasopressor therapy - in S3+ and S2+/S3 - patients. RRT: only renal replacement therapy, MV: only mechanical ventilation, TOS: triple organ support—combination of vasopressor therapy, MV and RRT. $\mathbf{b}$ Chord diagram representing administration of hydrocortisone $(\mathrm{HC})$ in septic shock. c Chord diagram representing the number of adjuvant vasopressor or inotropic agents on top of norepinephrine (NOR). Examined medications are epinephrine, vasopressin, dobutamine and milrinone. $\mathbf{d}$ Radar plot of the SOFA subscores: cardiac (SOFAcardio), neurological (SOFAcns), respiratory (SOFAresp), renal (SOFArenal), coagulation (SOFAcoag) and hepatic (SOFAliver)

Table 3 Univariate and multivariate analysis of variables associated with exclusion from the Sepsis-3 definition of septic shock

\begin{tabular}{lllr}
\hline & OR & $95 \%$ Cl & $p$ \\
\hline Univariate analysis & & & \\
Respiratory septic shock & 1.655 & $1.232-2.224$ & 0.001 \\
Abdominal septic shock & 0.501 & $0.352-0.712$ & $<0.001$ \\
Surgery on admission & 0.543 & $0.370-0.798$ & 0.002 \\
Medical admission reason & 2.128 & $1.576-2.872$ & $<0.001$ \\
Nicotine & 1.492 & $1.066-2.089$ & 0.02 \\
Alcohol abuse & 0.561 & $0.351-0896$ & 0.016 \\
Malignancy & 0.679 & $0.481-0.956$ & 0.027 \\
Hematological condition & 1.633 & $1.104-2.415$ & 0.014 \\
Chronic liver disease & 0.307 & $0.171-0.552$ & $<0.001$ \\
Chronic pulmonary disease & 1.461 & $1.031-2.069$ & 0.033 \\
Bacteremia & 0.607 & $0.424-0.870$ & 0.007 \\
Culture negative septic shock & 1.49 & $1.117-1.988$ & 0.007 \\
Multivariate analysis & & & \\
Respiratory septic shock & 1.485 & $1.056-2.089$ & 0.023 \\
Medical admission reason & 1.977 & $1.396-2.800$ & $<0.001$ \\
Chronic liver disease & 0.345 & $0.181-0.660$ & 0.001 \\
\hline
\end{tabular}

In our trial, we are essentially comparing 2 groups of patients that receive vasopressor therapy but that differ in lactate levels, in that sense that $\mathrm{S} 2+/ \mathrm{S} 3-$ patients never had a lactate level of $2 \mathrm{mmol} / \mathrm{l}$ or above during vasopressor therapy. This lactate criterion is the most important difference between the Sepsis-2 and Sepsis-3 consensus shock criteria [1, 2]. Lactate levels have been used as a marker of tissue hypoperfusion, with all limitations thereof since these are influenced by several mechanisms of lactate production (severity of shock, ischemia, administration of beta adrenergic medication, malignancies) and by lactate metabolism which is impaired in CLD [19]. Moreover, lactate levels are highly variable and must by consequence be interpreted as a snapshot in the course of the disease. Patients with higher lactate levels-and presumably with a higher degree of tissue hypoperfusion-in our cohort have higher severity scores and higher need for organ supportive therapy. This adds further support for the new septic shock definitions identifying a sicker cohort of patients with higher risk of mortality [1, 14-16, 20].

Three baseline characteristics were independently associated with not being included in the latest consensus 
definition of septic shock. First of all, excluded patients had more medical admission reasons compared to surgical admission reasons. We hypothesize that several medical admission reasons (like several inflammatory syndromes in hematological or immunosuppressed patients or after circulatory arrest that are nonetheless treated with antibiotics because there is doubt concerning an underlying infection) could be associated less with tissue hypoperfusion and lactate production compared with surgical admission reasons like peritonitis. This hypothesis is supported by the significant difference in abdominal infections and positive cultures between the $\mathrm{S} 2+/ \mathrm{S} 3-$ and S3+ patients. Secondly, more patients in the $\mathrm{S} 2+/ \mathrm{S} 3$ - group had a respiratory infection as cause for the septic shock. It is conceivable that these patients need more sedation to accommodate mechanical ventilation, and this may cause a higher need for vasopressor therapy which is not necessarily associated with shock and hypoperfusion. At last, the proportion of patients with CLD was three times higher in the S3+ group compared to the $\mathrm{S} 2+/ \mathrm{S} 3-$ group. This can be explained by reduced lactate clearance in patients with CLD, which may be more profound in the presence of sepsis-even in cases of mild liver disease. Patients with CLD also have higher ICU and hospital mortality compared to patients with normal hepatic function [19, 21-24] (Additional file 1: Tables S6-S8).

The lower mortality in the S2+/S3- group compared to the S3+group (31.6\% vs $55.3 \%$ ) is consistent with what other authors have reported $[1,3,14-16,20]$ while other authors report a similar mortality in both groups [4]. Mortality rates in our study were higher than what is found in the literature. This may be due to the high severity of the acute illness in our study population and to the high comorbidity burden (median CCI 4 in both groups) $[14,15,25]$.

When the new criteria for septic shock are applied for research purposes, the abovementioned patients are at risk of being excluded. By consequence, a different group of patients may be defined compared to trials using the Sepsis-2 criteria. This may introduce a gap in continuity of research in the field of septic shock. This is especially the case if the consensus definitions are systematically used for trial inclusion. However, when reviewing randomized controlled trials in septic shock, there is a vast heterogenicity in inclusion criteria which may have, not surprisingly, an effect on mortality figures [26]. This further complicates comparison between different interventional trials since the patients that are investigated differ to a greater or lesser extent. This effect is also seen in retrospective trials. When comparing several groups of patients with different criteria like need for vasopressor therapy, need for a certain lactate level or need for certain blood pressure targets-that can all be considered to be septic shock in one way or another, depending on the definition used-one notices a difference in number of patients pertaining to these groups but also a difference in mortality. This points out the important betweengroup variance $[17,18]$.

This was already noted by the authors of the third consensus conference definition who tried to create order in the existing chaos by introducing sound and new criteria to define septic shock, by selecting patients based on two objective criteria: clinically significant hypotension (and so need for vasopressor therapy) and tissue hypoperfusion (reflected by a rise in lactate above $2 \mathrm{mmol} / \mathrm{l}$ ) $[1,20]$. But no matter which criteria are used, in order to be able to comprehend, compare and discuss septic shock literature, it is important everyone speaks the same language, which means using a single set of diagnostic criteria worldwide.

Our study has some limitations: it has a monocentric retrospective design so the conclusions that apply to our tertiary patient population may not completely apply to other hospitals. The data used were primarily gathered for patient care, not for scientific research. Quality of the collected data depends, in no small amount, on correct prescriptions of medication or correct registration of parameters. Almost all data on baseline characteristics or comorbidity were collected at the time of admission to the ICU. The rate of missing data for the majority of variables was less than $5 \%$. For a few valuables like smoking and alcohol abuse, however, missing data rate could go as high as 14 to $20 \%$. Since the value of the Glasgow Coma Scale before patients are sedated is not always recorded, SOFA and APACHE II scores may be overestimated, but still reflect the high severity of illness in our cohort. The data are gathered entirely from the ICU Patient Data Management System. Since this is not used on normal wards or in the emergency department, data on vasopressor use or fluid therapy prior to admission on the ICU are not available.

\section{Conclusion}

One in five patients with septic shock according to the previous septic shock definition was no longer considered as such when the new Sepsis-3 septic shock criteria were applied. A medical admission reason, a respiratory focus of the septic shock and absence of chronic liver disease are associated with decreased probability of being defined as having septic shock according to the Sepsis-3 definition in our cohort.

\section{Abbreviations}

SOFA: Sequential Organ Failure Assessment; Hg: Mercury; SIRS: Systemic inflammatory response syndrome; qSOFA: Quick Sequential Organ Failure 
Assessment; ICU: Intensive care unit; COSARA: Computer-based surveillance and alerting of infections, antimicrobial resistance and antibiotic consumption in the ICU; CCl: Charlson comorbidity index; APACHE II: Acute Physiology and Chronic Health Evaluation II; CLD: Chronic liver disease; MV: Mechanical ventilation; RRT: Renal replacement therapy.

\section{Supplementary Information}

The online version contains supplementary material available at https://doi. org/10.1186/s13613-021-00942-1.

Additional file 1: Table S1. Distribution of the source of infection in S3+ and $\mathbf{S} 2+/ \mathrm{S} 3-$ patients. Figure $\mathbf{S 1}$. Distribution of the source of infection in S3+ and S2+/S3 - patients. Table S2. Baseline characteristics of the study population: extended. Table S3. Comorbidity characteristics: extended. Table S4. Organ support variables. Table S5. Maximal and minimal values of several organ dysfunction variables. Figure S2. Comparison of several organ dysfunction variable between $\mathrm{S} 3+$ and $\mathrm{S} 2+/ \mathrm{S} 3-$ Figure S3: Evolution of mortality of S3+ patients. Table S6. Comparison between septic shock patients with or without medical admission reason. Table S7. Comparison between septic shock patients with or without respiratory infection. Table S8. Comparison between septic shock patients with or without chronic liver disease. Table S9. Comparison in patients fulfilling Sepsis-3 criteria and patients not fulfilling Sepsis-3 criteria in the subgroup of patients with chronic liver disease. Figure S4. Proportion of $\mathrm{S} 3+$ patients and mortality in function of lactate levels. Figure S5. Propor tion of patients with chronic liver disease in the $\$ 2+/ \$ 3-$ and $\$ 3+$ group in function of lactate levels.

\section{Acknowledgements}

Not applicable.

\section{Authors' contributions}

JV had full access to all the data in the study and takes responsibility for the integrity of the data and the accuracy of the data analysis. JV, JDC and KC contributed to conception and design of the trial, and to analysis and interpretation of the data. JV, LDB and PD contributed to acquisition of the data. JV drafted the submitted article. JDC, LDB, PD and KC revised it critically. All authors have provided final approval of the version to be published.

\section{Funding}

No additional funding was obtained for this project.

\section{Availability of data and materials}

The datasets used during the current study are available from the corresponding author on reasonable request.

\section{Declarations}

\section{Ethics approval and consent to participate}

Approval for this study was obtained from the ethical committee of the Ghent University Hospital (EC/2016/0254) and the need for informed consent was waived.

\section{Competing interests}

The authors declare that they have no competing interest.

\section{Author details}

${ }^{1}$ Department of Intensive Care Medicine, University Hospital Gent, 2K12C. C. Heymanslaan 10, 9000 Gent, Belgium. ${ }^{2}$ Faculty of Medicine and Health Sciences, University Gent, Gent, Belgium.

Received: 31 March 2021 Accepted: 20 October 2021 Published online: 30 October 2021

\section{References}

1. Singer M, Deutschman CS, Seymour CW, Shankar-Hari M, Annane D, Bauer $\mathrm{M}$, et al. The third international consensus definitions for sepsis and septic shock (Sepsis-3). JAMA. 2016;315:801-10.

2. Levy MM, Fink MP, Marshall JC, Abraham E, Angus D, Cook D, et al. 2001 SCCM/ESICM/ACCP/ATS/SIS international sepsis definition conference. Crit Care Med. 2003:31:1250-6.

3. Fernando SM, Reardon PM, Rochwerg B, Shapiro NI, Yealy DM, Seely AJE, et al. Sepsis-3 septic shock criteria and associated mortality among infected hospitalized patients assessed by a rapid response team. Chest. 2018;154:309-16.

4. Henning DJ, Puskarich MA, SelfWH, Howel MD, Donnino MW, Yealy DM, et al. An emergency department validation of the SEP-3 sepsis and septic shock definitions and comparison with 1992 consensus definitions. Ann Emerg Med. 2017;70:544-52.

5. Raith EP, Udy AA, Bailey M, McGloughlin S, Maclsaac C, Bellomo R, et al. Prognostic accuracy of the SOFA score, SIRS criteria, and qSOFA score for in-hospital mortality among adults with suspected infection admitted to the intensive care unit. JAMA. 2017;317:290-300.

6. Freund $Y$, Lemachatti N, Krastinova E, van Laer M, Claessens Y, Avondo A, et al. Prognostic accuracy of sepsis-3 criteria for in-hospital mortality among patients with suspected infection presenting to the emergency department. JAMA. 2017;317:301-8.

7. Fang $X$, Wang Z, Yang J, Cai H, Yao Z, Li K, et al. Clinical evaluation of sepsis-1 and sepsis-3 in the ICU. Chest. 2018;153:1169-76.

8. De Bus L, Diet G, Gadeyne B, Leroux-Roels I, Claeys G, Steurbaut K, et al. Validity analysis of a unique infection surveillance system in the intensive care unit by analysis of a data warehouse built through a workflowintegrated software application. J Hosp Infect. 2014;87:159-64.

9. De Bus L, Gadeyne B, Steen J, Boelens J, Claeys G, Benoit D, et al. A complete and multifaceted overview of antibiotic use and infection diagnosis in the intensive care unit: results from a prospective four-year registration. Crit Care. 2018;22:241.

10. Rhodes A, Evans LE, Alhazzani W, Levy MM, Antonelli M, Ferrer R, et al. Surviving sepsis campaign: international guidelines for management of sepsis and septic shock: 2016. Intensive Care Med. 2017:43:304-77.

11. Charlson ME, Pompei P, Ales KL, Mackenzie CR. A new method of classifying prognostic comorbidity in longitudinal studies: development and validation. J Chronic Dis. 1987:40:73-83.

12. Vincent JL, de Mendonça A, Cantraine F, Moreno R, Takala J, Suter PM, et al. Use of the SOFA score to assess the incidence of organ dysfunction/ failure in intensive care units: results of a multicenter prospective study. Working group on "sepsis-related problems" of the European Society of Intensive Care Medicine. Crit Care Med. 1998;26:1793-800.

13. Knaus WA, Draper EA, Wagner DP, Zimmerman JE. APACHE II: a severity of disease classification system. Crit Care Med. 1985;13:181-829.

14. Ryoo SM, Kang GU, Shin TG, Hwang SY, Kim K, Jo YH, et al. Clinical outcome comparison of patients with septic shock defined by the new sepsis-3 criteria and by previous criteria. J Thorac Dis. 2018;10:845-53.

15. Sterling SA, Puskarich MA, Glass AF, Guirgis F, Jones AE. The impact of the sepsis-3 septic shock definition on previously defined septic shock patients. Crit Care Med. 2017;45:1436-42.

16. Driessen RGH, van de Poll MCG, Mol MF, Van Mook WNKA, Schnabel RM. The influence of a change in septic shock definitions on intensive care epidemiology and outcome: comparison of sepsis-2 and sepsis-3 definitions. Infect Dis (Lond). 2018;50:207-13.

17. SepNet Critical Care Trials Group. Incidence of severe sepsis and septic shock in German intensive care units: the prospective, multicentre INSEP study. Intensive Care Med. 2016;42:1980-9.

18. Estenssoro E, Kanoore Edul VS, Loudet Cl, Osatnik J, Rios FG, Vazquez DN, et al. Predictive validity of sepsis-3 definitions and sepsis outcomes in critically ill patients: a cohort study in 49 ICUs in argentina. Crit Care Med. 2018;46:1276-83.

19. Kraut JA, Madias NE. Lactic acidosis. N Engl J Med. 2014:371:2309-19.

20. Shankar-Hari M, Phillips GS, Levy ML, Seymour CW, Liu VX, Deutschman CS, et al. Developing a new definition and assessing new clinical criteria for septic shock: for the third international consensus definitions for sepsis and septic shock (sepsis-3). JAMA. 2016;315:775-87.

21. Dugas AF, Mackenhauer J, Salciccioli JD, Cocchi MN, Gautam S, Donnino MW. Prevalence and characteristics of nonlactate and lactate expressors in septic shock. J Crit Care. 2012:27:344-50. 
22. Jeppesen JB, Mortensen C, Bendtsen F, Moller S. Lactate metabolism in chronic liver disease. Scand J Lab Invest. 2013;73:293-9.

23. Galbois A, Aegerter P, Martel-Samb P, Housset C, Thabut D, Offenstadt $G$, et al. Improved prognosis of septic shock in patients with cirrhosis: a multicenter study. Crit Care Med. 2014;42:1666-75.

24. De Waele JJ, Lipman J, Sakr Y, Marshall JC, Vanhems P, Groba CB, et al. Abdominal infections in the intensive care unit: characteristics, treatment and determinants of outcome. BMC Infect Dis. 2014;14:420.

25. Ladha KS, Zhao K, Quraishi SA, Kurth T, Eikermann M, Kaafarani HMA, et al. The Deyo-Charlson and Elixhauser-van Walraven comorbidity indices as predictors of mortality in critically ill patients. BMJ Open. 2015;5:e008990.
26. De Grooth HJ, Postema J, Loer SA, Parienti JJ, Oudemans-Van Straaten HM, Girbes AR. Unexplained mortality differences between septic shock trials: a systematic analysis of population characteristics and controlgroup mortality rates. Intensive Care Med. 2018;44:311-22.

\section{Publisher's Note}

Springer Nature remains neutral with regard to jurisdictional claims in published maps and institutional affiliations.

\section{Submit your manuscript to a SpringerOpen ${ }^{\circ}$ journal and benefit from:}

- Convenient online submission

- Rigorous peer review

- Open access: articles freely available online

- High visibility within the field

- Retaining the copyright to your article

Submit your next manuscript at $\boldsymbol{\nabla}$ springeropen.com 